\title{
A study of the temperature dependence of bienzyme systems and enzymatic chains
}

\author{
N. V. KOTOV $\dagger$, R. E. BAKER $\ddagger *$, D. A. DAWIDOV $\dagger$, K. V. PLATOV $\dagger$, N. V. VALEYEV $\| \#$,
} A. I. SKORINKIN $\uparrow \S$ and P. K. MAINI†\|

$\dagger$ Laboratory of Biophysics and Bionics, Physics Department, Kazan State University, Kazan 420018, Russia

$\ddagger$ Centre for Mathematical Biology, Mathematical Institute, University of Oxford, 24-29 St. Giles, Oxford OX1 3LB, UK

ๆDepartment of Biochemistry, University of Oxford, South Parks Road, Oxford OX1 3QU, UK

$\S$ Kazan Institute of Biochemistry and Biophysics, Kazan State University, Russian Academy of Sciences, Kazan 420018, Russia

||Department of Biochemistry, Oxford Centre for Integrative Systems Biology, University of Oxford, South Parks Road, Oxford OX1 3QU, UK

\#Systems Biology Lab, Department of Engineering, University of Leicester, University Road, Leicester LE1 7RH, UK

(Received 6 October 2006; revised 16 March 2007; in final form 28 March 2007)

\begin{abstract}
It is known that most enzyme-facilitated reactions are highly temperature dependent processes. In general, the temperature coefficient, $Q_{10}$, of a simple reaction reaches 2.0-3.0. Nevertheless, some enzyme-controlled processes have much lower $Q_{10}$ (about 1.0), which implies that the process is almost temperature independent, even if individual reactions involved in the process are themselves highly temperature dependent. In this work, we investigate a possible mechanism for this apparent temperature compensation: simple mathematical models are used to study how varying types of enzyme reactions are affected by temperature. We show that some bienzyme-controlled processes may be almost temperature independent if the modules involved in the reaction have similar temperature dependencies, even if individually, these modules are strongly temperature dependent. Further, we show that in non-reversible enzyme chains the stationary concentrations of metabolites are dependent only on the relationship between the temperature dependencies of the first and last modules, whilst in reversible reactions, there is a dependence on every module. Our findings suggest a mechanism by which the metabolic processes taking place within living organisms may be regulated, despite strong variation in temperature.
\end{abstract}

Keywords: Bienzyme systems; Reversible reactions; Non-reversible reactions; Temperature dependence; Mathematical modelling

AMS Subject Classification: 92B99; 92E20

\section{Introduction}

Many important processes in the living cell are based on networks of enzyme-controlled chemical reactions where the enzyme activity is strongly temperature dependent; see, for example, $[13,19,20,23,27]$. A commonly used parameter to measure the temperature dependence of a chemical reaction is $Q_{10}$, which is defined as the change in reaction rate with a $10^{\circ} \mathrm{K}$ change in temperature. For example, a $Q_{10}$ value of 2.0 would imply a two-fold

*Corresponding author. Email: ruth.baker@maths.ox.ac.uk

Computational and Mathematical Methods in Medicine 
increase in reaction rate with a $10^{\circ} \mathrm{K}$ change in temperature. It is generally accepted that in the majority of cases, $Q_{10}$ lies between 2.0 and 3.0 [22] although it should be noted that $Q_{10}$ is not necessarily constant over the entire temperature range [37].

In contrast, there are some enzyme-controlled processes which display a much higher temperature dependence, with $Q_{10}>3.0$; see, for example, [2,8,25,35]; and some enzymecontrolled processes which are almost temperature independent (with $Q_{10} \approx 1.0$ ); see, for example, $[16,28,36]$. In these temperature independent processes it may be the case that the individual reactions display a strong temperature dependence [19,20,23,27,31,37], but that their combined action contains some adapting step so that the resulting process is virtually temperature independent.

There have been numerous studies on the effects of temperature change upon living organisms: how metabolism may be regulated via enzyme and protein adaptations $[31-34,38,44]$, but to our knowledge there has been little theoretical investigation from the traditional enzyme kinetics viewpoint into the mechanisms via which enzyme-controlled reactions compensate for temperature changes [9,11,17,40-42].

The main question we address in this study is how temperature changes can influence networks of enzyme reactions. There are many different and very complex mechanisms for temperature control developed during evolution, especially in multicellular organisms. However, in this work, we suggest a mechanism via which a simple system (such as a protein network) can operate as a temperature independent module even if "parts" of that module are temperature dependent.

Initially, we start with a model bienzyme system and show that the steady state concentrations of reacting substrates are dependent on the ratios of the active enzyme concentrations and rate constants. If the rate constants satisfy certain relationships then we show that the system can be almost temperature independent. We illustrate this by considering some commonly occurring bienzyme systems in more detail. In the second part of this article, we apply similar techniques to chains of reversible and non-reversible reactions.

\section{Initial studies: simple bienzyme modules}

In this section, we begin our study by considering the simplest kind of bienzyme modules. In each case, an initial substrate $\left(S_{1}\right)$ will react with an initial enzyme $\left(E_{1}\right)$ to produce a second substrate $\left(S_{2}\right)$. This second substrate will react with a second enzyme $\left(E_{2}\right)$ to produce either a product $(P)$ or the initial substrate $\left(S_{1}\right)$. We illustrate our findings with some commonly occurring metabolic reactions.

\subsection{A non-reversible bienzyme module}

The most simple non-reversible bienzyme module can be written as follows:

$$
\begin{aligned}
& S_{1}+E_{1} \underset{k_{-1}}{\stackrel{k_{1}}{\rightleftharpoons}} C_{1} \stackrel{k_{2}}{\longrightarrow} S_{2}+E_{1}, \\
& S_{2}+E_{2} \underset{k_{-3}}{\stackrel{k_{3}}{\rightleftharpoons}} C_{2} \stackrel{k_{4}}{\longrightarrow} P+E_{2} .
\end{aligned}
$$


Using the Law of Mass Action the module can be described simply by the chemical equations shown below:

$$
\begin{gathered}
\frac{\mathrm{d} s_{2}}{\mathrm{~d} t}=-k_{3} s_{2} e_{2}+k_{-3} c_{2}+k_{2} c_{1}, \\
\frac{\mathrm{d} c_{1}}{\mathrm{~d} t}=k_{1} s_{1} e_{1}-\left(k_{-1}+k_{2}\right) c_{1}, \\
\frac{\mathrm{d} c_{2}}{\mathrm{~d} t}=k_{3} s_{2} e_{2}-\left(k_{-3}+k_{4}\right) c_{2}, \\
\frac{\mathrm{d} e_{1}}{\mathrm{~d} t}=-k_{1} s_{1} e_{1}+\left(k_{-1}+k_{2}\right) c_{1}, \\
\frac{\mathrm{d} e_{2}}{\mathrm{~d} t}=-k_{3} s_{2} e_{2}+\left(k_{-3}+k_{4}\right) c_{2}, \\
\frac{\mathrm{d} p}{\mathrm{~d} t}=k_{4} c_{2},
\end{gathered}
$$

where $s_{1}=\left[S_{1}\right]$, the concentration of $S_{1}$, etc. Here, we are assuming that the concentration of $S_{1}$ is fixed by other enzyme systems which we will not explicitly model. We assume initial conditions of the form

$$
\left(s_{1}, s_{2}, c_{1}, c_{2}, e_{1}, e_{1}, p\right)=\left(s_{1}^{0}, 0,0,0, e_{1}^{A}, e_{2}^{A}, 0\right) .
$$

The equations for $c_{i}$ and $e_{i}(i=1,2)$ can be added and integrated to give the result

$$
\begin{aligned}
& c_{1}(t)+e_{1}(t)=e_{1}^{A}, \\
& c_{2}(t)+e_{2}(t)=e_{2}^{A},
\end{aligned}
$$

where $e_{i}^{A}$ is the active concentration of enzyme $E_{i}$ for $i=1,2$. It should be noted that the active concentrations of enzymes can be regulated by factors such as temperature, $\mathrm{pH}$, covalent modification and external concentrations of activators and inhibitors [1,7]. Therefore, a sudden change in temperature may cause a shift in the concentration of active enzyme.

Assuming that the system is in a steady state we have

$$
c_{1}=\frac{s_{1} e_{1}^{A}}{K_{1}+s_{1}} \quad \text { and } \quad c_{2}=\frac{s_{2} e_{2}^{A}}{K_{2}+s_{2}},
$$

where $K_{1}$ and $K_{2}$ are the standard Michaelis-Menten constants given by

$$
K_{1}=\frac{k_{-1}+k_{2}}{k_{1}} \quad \text { and } \quad K_{2}=\frac{k_{-3}+k_{4}}{k_{3}} .
$$

Thus, the steady state concentration of $S_{2}$, given by $s_{2}^{*}$, satisfies the equation

$$
s_{2}^{*}=\frac{K_{2}}{\frac{A_{2}}{A_{1}}\left(\frac{K_{1}}{s_{1}^{*}}+1\right)-1},
$$

where $s_{1}^{*}$ is the steady state concentration of $S_{1}$ and $A_{i}=k_{2 i} e_{i}^{A}$ for $i=1,2$. The concentration of $S_{1}$ is held fixed in this example and so we have $s_{1}^{*}=s_{1}^{0}$. Assuming that $s_{1}^{0} \gg K_{1}$ we have 
an approximate stationary solution for $S_{2}$ given by

$$
s_{2}^{*}=\frac{K_{2}}{\frac{A_{2}}{A_{1}}-1} \text {. }
$$

Thus, under the above assumptions, the stationary concentration of $S_{2}$ is a simple function of the ratio of the active enzyme concentrations and the rate constants. Note that in order for a feasible steady state to exist, that is for $s_{2}^{*}>0$, we must have $A_{2}>A_{1}$.

Figure 1 shows how the steady state concentration of $S_{2}$ depends on the rate constants $k_{2}$ and $k_{4}$. As expected, as $k_{2}$ is increased $s_{2}^{*}$ increases and vice-versa for $k_{4}$. The plots are produced using rates within the typical range for a substrate-enzyme reaction.

(a) Change in $s_{2}^{*}$ with $k_{2}$

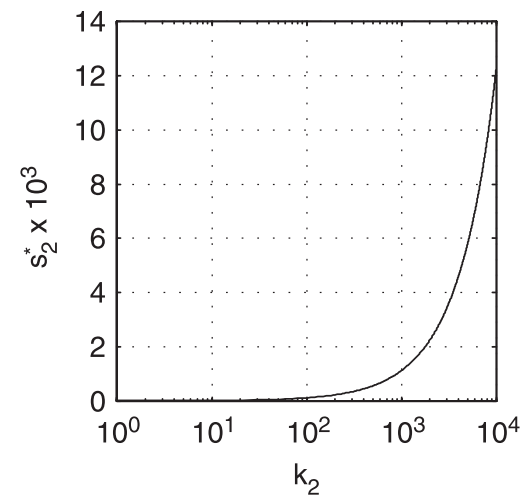

(c) Change in $\mathrm{s}_{2}^{*}$ with $\mathrm{k}_{2}$

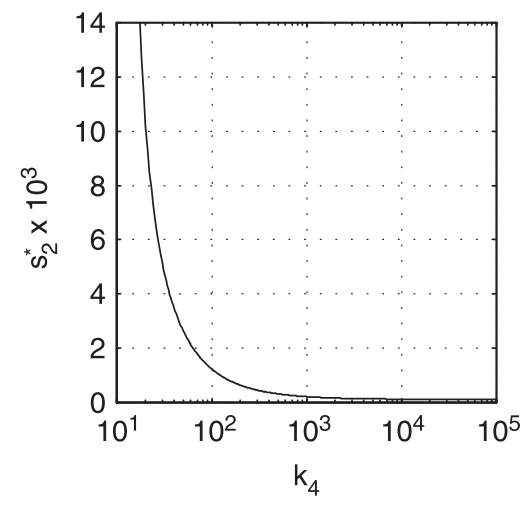

(b) Change in $s_{2}^{*}$ with $e_{1}^{A}$

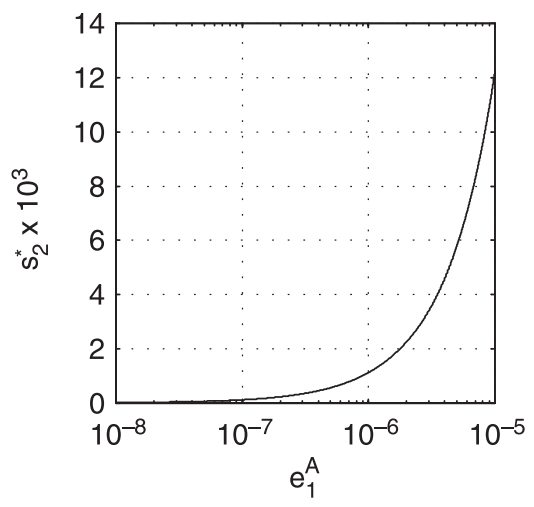

(d) Change in $s_{2}^{*}$ with $e_{2}^{A}$

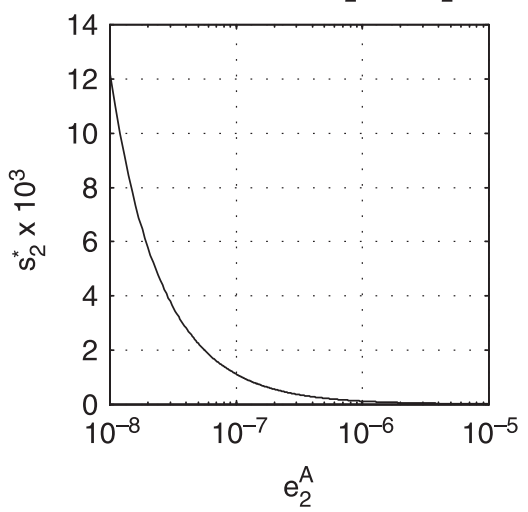

Figure 1. The change in the steady state concentration $s_{2}^{*}$, given by equation (13), whilst $k_{2}, k_{4}$ and $e_{1}^{A}, e_{2}^{A}$ are varied. In each plot, the rate constant/enzyme activity parameter is shown on a $\log _{10}$ scale. Parameters are as follows: (a) $k_{1}=10^{6} \mathrm{M}^{-1} \mathrm{~s}^{-1}, k_{-1}=10^{2} \mathrm{~s}^{-1}, k_{3}=10^{5} \mathrm{M}^{-1} \mathrm{~s}^{-1}, k_{-3}=10^{3} \mathrm{~s}^{-1}, k_{4}=10^{4} \mathrm{~s}^{-1}, e_{1}^{A}=10^{-7} \mathrm{M}$ and $e_{2}^{A}=10^{-6} \mathrm{M}$; (b) $k_{1}=10^{6} \mathrm{M}^{-1} \mathrm{~s}^{-1}, \quad k_{-1}=10^{2} \mathrm{~s}^{-1}, \quad k_{2}=10^{2} \mathrm{~s}^{-1}, \quad k_{3}=10^{5} \mathrm{M}^{-1} \mathrm{~s}^{-1}, \quad k_{-3}=10^{3} \mathrm{~s}^{-1}, \quad k_{4}=10^{4} \mathrm{~s}^{-1}$ and $e_{2}^{A}=10^{-6} \mathrm{M}$; (c) $k_{1}=10^{6} \mathrm{M}^{-1} \mathrm{~s}^{-1}, k_{-1}=10^{2} \mathrm{~s}^{-1}, k_{2}=10^{2} \mathrm{~s}^{-1}, k_{3}=10^{5} \mathrm{M}^{-1} \mathrm{~s}^{-1}, k_{-3}=10^{3} \mathrm{~s}^{-1}, e_{1}^{A}=$ $10^{-7} \mathrm{M}$ and $e_{2}^{A}=10^{-6} \mathrm{M}$; (d) $k_{1}=10^{6} \mathrm{M}^{-1} \mathrm{~s}^{-1}, k_{-1}=10^{2} \mathrm{~s}^{-1}, k_{2}=10^{2} \mathrm{~s}^{-1}, k_{3}=10^{5} \mathrm{M}^{-1} \mathrm{~s}^{-1}, k_{-3}=10^{3} \mathrm{~s}^{-1}$, $k_{4}=10^{4} \mathrm{~s}^{-1}$ and $e_{1}^{A}=10^{-7} \mathrm{M}$. 
2.1.1 Example module. The above module and the corresponding biological assumptions can be illustrated by considering a module involved in the regulation of cAMP concentration. The metabolic module can be described as follows:

$$
\begin{gathered}
\mathrm{ATP}+\mathrm{AC} \underset{k_{-1}}{\stackrel{k_{1}}{\rightleftharpoons}} \mathrm{ATP}-\mathrm{AC} \stackrel{k_{2}}{\longrightarrow} \mathrm{cAMP}+\mathrm{AC}, \\
\mathrm{cAMP}+\mathrm{PDE} \underset{k_{-3}}{\stackrel{k_{3}}{\rightleftharpoons}} \mathrm{cAMP}-\mathrm{PDE} \stackrel{k_{4}}{\longrightarrow} \mathrm{MN}+\mathrm{PDE},
\end{gathered}
$$

where AC is Adenylate cyclase, the enzyme transforming Adenosintriphosphate (ATP) to cyclic Adenosinmonophosphate (cAMP) and PDE is Phosphodiestherase, the enzyme transforming cAMP to Mononucleotide (MN).

From Refs. [15,18,29], we have that:

$$
[\mathrm{ATP}]^{0}>10^{-3} \mathrm{M} \gg 10^{-4} \mathrm{M} \geq \frac{k_{-1}+k_{2}}{k_{1}}=K_{1},
$$

where $[\mathrm{ATP}]^{0}$ is the initial concentration of ATP. Applying equation (13) we can deduce that the stationary concentration of cAMP is given approximately by

$$
[\mathrm{cAMP}]^{*}=\frac{K_{2}}{\frac{k_{4}[\mathrm{PDE}]^{A}}{k_{2}[\mathrm{AC}]^{4}}-1}
$$

where $[\mathrm{PDE}]^{A}$ is the active concentration of PDE and $K_{2}=\left(k_{-3}+k_{4}\right) / k_{3}$. Thus, the stationary concentration of cAMP is a function of the ratio of AC and PDE activities and the rate constants.

Whilst we expect the active enzyme concentrations to depend on temperature, there will also be external factors that may change the number of active receptors. In the metabolic module considered above, changes in AC receptor concentration may be caused by the presence of adrenalin and the Cholera toxin [1].

\subsection{A reversible bienzyme system}

The most simple reversible bienzyme reaction may be written in the following way:

$$
\begin{aligned}
& S_{1}+E_{1} \underset{k_{-1}}{\stackrel{k_{1}}{\rightleftharpoons}} C_{1} \stackrel{k_{2}}{\longrightarrow} S_{2}+E_{1}, \\
& S_{2}+E_{1}^{\prime} \underset{k_{-3}^{\prime}}{\stackrel{k_{3}^{\prime}}{\rightleftharpoons}} C_{1}^{\prime} \stackrel{k_{2}^{\prime}}{\longrightarrow} S_{1}+E_{1}^{\prime} .
\end{aligned}
$$

The end product is simply the initial substrate and hence the system differs little from that of section 1 except that here we do not assume that $S_{1}$ is held fixed. Using exactly the same method, the steady state concentration of $S_{2}$ is found to be

$$
s_{2}^{*}=\frac{K_{2}^{\prime}}{\frac{A_{1}^{\prime}}{A_{1}}\left(\frac{K_{1}}{s_{1}^{*}}+1\right)-1},
$$


where

$$
K_{1}=\frac{k_{-1}+k_{2}}{k_{1}}, \quad K_{2}^{\prime}=\frac{k_{-3}^{\prime}+k_{2}^{\prime}}{k_{3}^{\prime}}, \quad A_{1}=k_{2} e_{1}^{A} \quad \text { and } \quad A_{1}^{\prime}=k_{2}^{\prime} e_{1}^{\prime A}
$$

When the system is in a steady state we have $s_{1}(t)+s_{2}(t)=s_{1}(0)=s_{1}^{0}$ (this can also be seen by noting that substrate is conserved). In this case, we have an equation for $s_{2}^{*}$ :

$$
s_{2}^{*}=\frac{K_{2}^{\prime}}{\frac{A_{1}^{\prime}}{A_{1}}\left(\frac{K_{1}}{s_{1}^{0}-s_{2}^{*}}+1\right)-1},
$$

which can be solved to give

$$
s_{2}^{*}=\frac{\left[\frac{A_{1}^{\prime}}{A_{1}}-1\right] s_{1}^{0}+\left[K_{2}^{\prime}+\frac{A_{1}^{\prime}}{A_{1}} K_{1}\right] \pm \sqrt{\left(\left[\frac{A_{1}^{\prime}}{A_{1}}-1\right] s_{1}^{0}+\left[K_{2}^{\prime}+\frac{A_{1}^{\prime}}{A_{1}} K_{1}\right]\right)^{2}-4\left[\frac{A_{1}^{\prime}}{A_{1}}-1\right] s_{1}^{0} K_{2}^{\prime}}}{2\left(\frac{A_{1}^{\prime}}{A_{1}}-1\right)} .
$$

We note that, since the system is conservative, we have non-zero steady states for both $S_{1}$ and $S_{2}$ even without a supply of $S_{1}$ from external factors. If the concentration of $S_{1}$ is changed, the system will settle to a new steady state with an adjusted value of $s_{1}^{0}$.

Figure 2 shows plots of the steady state concentration of $S_{2}$ as the rate constants $k_{2}$ and $k_{4}$ are varied, in both the cases $A_{1}^{\prime} / A_{1} \lessgtr 1$. Although it appears as if there are two solutions for $s_{2}^{*}$ in each plot in figure 2 , in fact only one of these will be feasible. In the case that $A_{1}^{\prime} / A_{1}>1$ the positive square root will always give $s_{2}^{*}>s_{1}^{0}$ which contradicts the initial conditions. In the case that $A_{1}^{\prime} / A_{1}<1$ we must take the square root which results in a positive solution for $s_{2}^{*}$. In each of the cases $A_{1}^{\prime} / A_{1} \lessgtr 1$, we see results similar to the non-reversible case: increasing $k_{2}$ increases $s_{2}^{*}$ and vice versa for $k_{4}$. Similar results hold for $e_{1}^{A}$ and $e_{2}^{A}$ but we do not show them here.

2.2.1 An example module. A widespread process that takes place in many living cells is phosphorylation/dephosphorylation. Let us consider a bienzyme module that covalently modifies some protein conformation by phosphorylation and dephosphorylation. In this module Protein Kinase (PK) phosphorylates a protein (P) using an ATP molecule as a source of phosphate. Phosphoproteinphosphatase $(\mathrm{PH})$ dephosphorylates the protein:

$$
\begin{aligned}
& \mathrm{P}+\mathrm{PK} \underset{k_{-1}}{\stackrel{k_{1}}{\rightleftharpoons}} \mathrm{P}-\mathrm{KP} \stackrel{k_{2}}{\longrightarrow} \mathrm{P}_{p}+\mathrm{PK}, \\
& \mathrm{P}_{p}+\mathrm{PH} \underset{k_{-3}^{\prime}}{\stackrel{k_{3}^{\prime}}{\rightleftharpoons}} \mathrm{P}_{p-\mathrm{PH}} \stackrel{k_{2}^{\prime}}{\longrightarrow} \mathrm{P}+\mathrm{PH} .
\end{aligned}
$$

Assuming that the system is in a steady state and using the method of section 2.2, we can write down an equation for the steady state level of phosphorylated protein:

$$
0=k_{2}[\mathrm{PK}]^{A} \frac{\left(P_{0}-\left[\mathrm{P}_{p}\right]\right)}{K_{1}+\left(P_{0}-\left[\mathrm{P}_{p}\right]\right)}-k_{4}[\mathrm{PH}]^{A} \frac{\left[\mathrm{P}_{p}\right]}{K_{2}^{\prime}+\left[\mathrm{P}_{p}\right]},
$$


(a) Change in $s_{2}^{*}$ with $k_{2}-A_{1}^{\prime} / A_{1}<1$

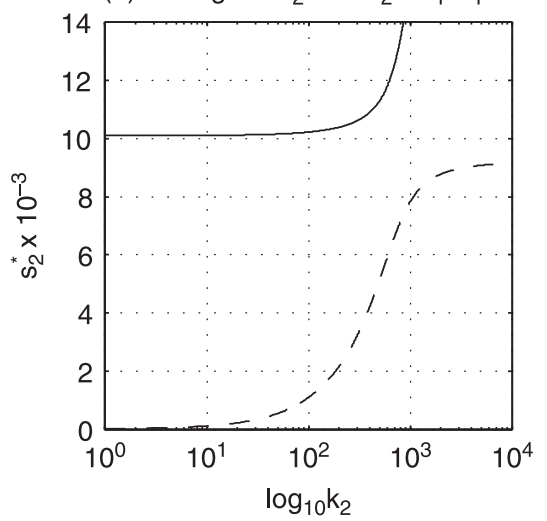

(c) Change in $s_{2}^{\star}$ with $k_{4}-A_{1}^{\prime} / A_{1}<1$

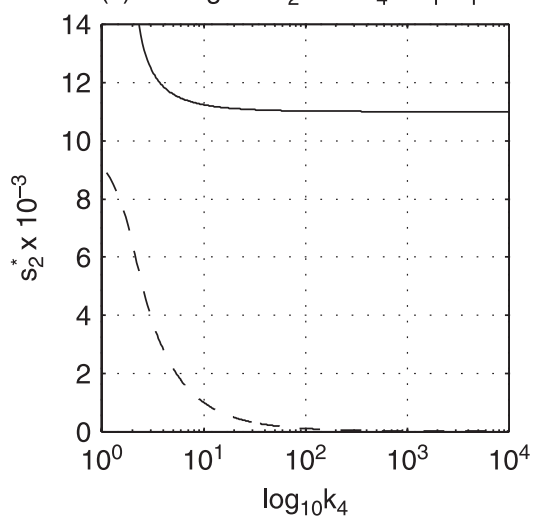

(b) Change in $s_{2}^{*}$ with $k_{2}-A_{1}^{\prime} / A_{1}>1$

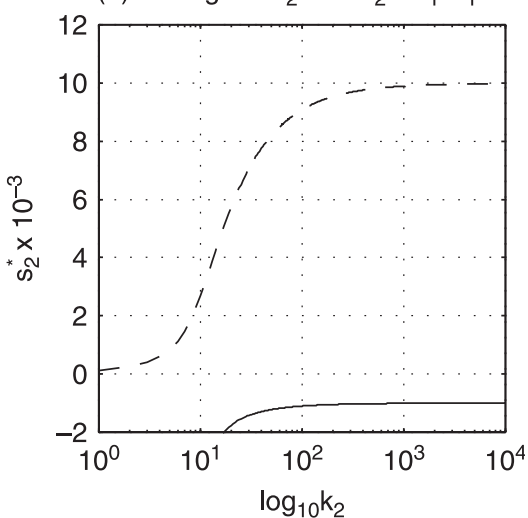

(d) Change in $s_{2}^{*}$ with $k_{4}-A_{1}^{\prime} / A_{1}>1$

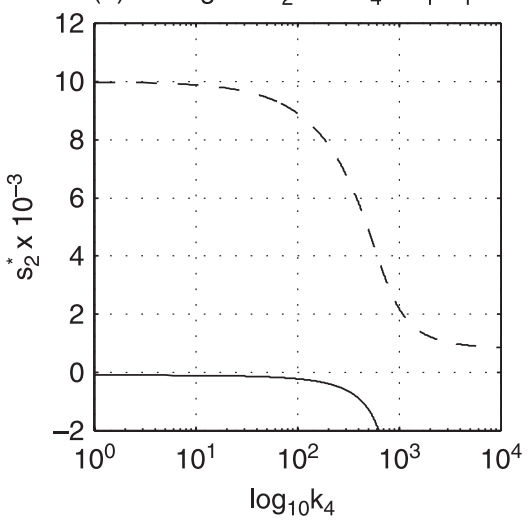

Figure 2. The change in the steady state concentration $s_{2}^{*}$ in the closed system whilst $k_{2}$ and $k_{4}$ are varied. $s_{2}^{*}$ is given by equation (22). In each plot, the rate constant is shown on a $\log _{10}$ scale, the solid line denotes the solution using the positive square root and the dashed line denotes the solution using the negative square root. Parameters are as follows: (a) $k_{1}=10^{6} \mathrm{M}^{-1} \mathrm{~s}^{-1}, k_{-1}=10^{2} \mathrm{~s}^{-1}, k_{3}=10^{5} \mathrm{M}^{-1} \mathrm{~s}^{-1}, k_{-3}=10^{4} \mathrm{~s}^{-1}, k_{4}=10^{3} \mathrm{~s}^{-1}, e_{1}^{A}=10^{-7} \mathrm{M}$ and $e_{2}^{A}=10^{-6} \mathrm{M}$; (b) $k_{1}=10^{4} \mathrm{M}^{-1} \mathrm{~s}^{-1}, k_{-1}=10^{4} \mathrm{~s}^{-1}, k_{3}=10^{5} \mathrm{M}^{-1} \mathrm{~s}^{-1}, k_{-3}=10^{2} \mathrm{~s}^{-1}, k_{4}=10^{0} \mathrm{~s}^{-1}, e_{1}^{A}=10^{-6} \mathrm{M}$ and $e_{2}^{A}=10^{-7} \mathrm{M}$; (c) $k_{1}=10^{6} \mathrm{M}^{-1} \mathrm{~s}^{-1}, k_{-1}=10^{3} \mathrm{~s}^{-1}, k_{3}=10^{1} \mathrm{M}^{-1} \mathrm{~s}^{-1}, k_{-3}=10^{5} \mathrm{~s}^{-1}, k_{4}=10^{3} \mathrm{~s}^{-1}, e_{1}^{A}=$ $10^{-7} \mathrm{M}$ and $e_{2}^{A}=10^{-6} \mathrm{M}$; (d) $k_{1}=10^{4} \mathrm{M}^{-1} \mathrm{~s}^{-1}, k_{-1}=10^{2} \mathrm{~s}^{-1}, k_{2}=10^{3} \mathrm{~s}^{-1}, k_{-3}=10^{2} \mathrm{~s}^{-1}, k_{4}=10^{6} \mathrm{~s}^{-1}, e_{1}^{A}=$ $10^{-6} \mathrm{M}$ and $e_{2}^{A}=10^{-7} \mathrm{M}$.

where $K_{1}$ and $K_{2}^{\prime}$ are as in equation $(20),[\mathrm{PK}]^{A},[\mathrm{PH}]^{A}$ are the active concentrations of $\mathrm{PK}$ and $\mathrm{PH}$ molecules, respectively, and $P^{0}=[\mathrm{P}]+\left[\mathrm{P}_{p}\right]$. Solving the above we see that

$$
\left[\mathrm{P}_{p}\right]^{*}=\frac{\left[\frac{A_{1}^{\prime}}{A_{1}}-1\right] P^{0}+\left[K_{2}^{\prime}+\frac{A_{1}^{\prime}}{A_{1}} K_{1}\right] \pm \sqrt{\left(\left[\frac{A_{1}^{\prime}}{A_{1}}-1\right] P^{0}+\left[K_{2}^{\prime}+\frac{A_{1}^{\prime}}{A_{1}} K_{1}\right]\right)^{2}-4\left[\frac{A_{1}^{\prime}}{A_{1}}-1\right] P^{0} K_{2}^{\prime}}}{2\left(\frac{A_{1}^{\prime}}{A_{1}}-1\right)}
$$

where $K_{1}$ and $K_{2}^{\prime}$ are as in equation (20), $A_{1}=k_{2}[\mathrm{PK}]^{A}$ and $A_{1}^{\prime}=k_{2}^{\prime}[\mathrm{PH}]^{A}$. 


\subsection{Discussion: the temperature regulation of bienzyme modules}

From the results of the previous sections, we deduce that under certain conditions the steady state concentration of $S_{2}$ is dependent on the Michaelis-Menten constants, and the ratios between the rate constants and the active concentrations of enzymes. We now discuss a possible mechanism for temperature compensation. To begin, we discuss the temperature dependence of the rate constants and enzyme activities.

2.3.1 Dependence of rate constants on temperature. If we examine only temperature ranges where protein denaturation is negligible, then the Arrhenius equation predicts the following dependence of rate constant upon temperature:

$$
k=B \exp \left(-\frac{E}{R T}\right)
$$

where $B$ is a constant, $E$ is the activation energy of the reaction, $R$ is the gas constant and $T$ is the temperature (in Kelvin). Suppose that a simple substrate-enzyme reaction $S+E \rightarrow P$ has rate constants $k_{ \pm 1}, k_{2}$. Then the associated Michaelis Menten rate constant will be given by

$$
\begin{gathered}
K=\frac{B_{-1} \exp \left(-\frac{E-1}{R T}\right)+B_{2} \exp \left(-\frac{E_{2}}{R T}\right)}{B_{1} \exp \left(-\frac{E_{1}}{R T}\right)} \\
\Rightarrow \quad K=\frac{B_{-1}}{B_{1}} \exp \left[-\frac{\left(E_{-1}-E_{1}\right)}{R T}\right]+\frac{B_{2}}{B_{1}} \exp \left[-\frac{\left(E_{2}-E_{1}\right)}{R T}\right] .
\end{gathered}
$$

Assuming that the activation energies, $E_{i}$, are similar then $K$ will be virtually temperature independent.

Figure 3(a) shows a plot of the temperature dependence of a rate constant $k$ according to the Arrhenius equation. Figure 3(b) shows a plot of a Michaelis-Menten rate constant $K$ with each of the constituent rate constants having Arrhenius temperature dependence. We see from figure 3(b) that a $10^{\circ} \mathrm{K}$ change in temperature results in a change in $K$ of about $1 \%$, demonstrating that with similar activation energies the Michaelis-Menten constant, and hence $Q_{10}$, will be virtually temperature independent.

There are several examples in the literature where this phenomenon of temperature independence of the Michaelis-Menten rate constants has been observed. For temperature independence of the parameters in the ATP/AC reaction of section 2.1.1 see, for example, $[30,43]$ and for a phosphorylation reaction (as in section 2.2.1) see [31].

2.3.2 Dependence of $s_{2}^{*}$ upon temperature. As was shown in section 2.3.1, if the activation energies for two reactions are similar, or if the proteins are not labile with respect to temperature, then the Michaelis-Menten constants are only weakly temperature dependent. In this case the temperature dependence of $s_{2}^{*}$ comes from the ratio of $A_{1}=k_{2} e_{1}^{A}$ and $A_{2}=k_{4} e_{2}^{A}$. We expect the rate constants $k_{2}$ and $k_{4}$ to follow the Arrhenius equation and $e_{1}^{A}$ and $e_{2}^{A}$ to display a bell-shaped temperature dependence [3,10,12-14].

Figure 4 shows the expected temperature dependence of $s_{2}^{*}$ in the non-reversible reaction given by equation (1) when $A_{1}=k_{2} e_{1}^{A}$ and $A_{2}=k_{4} e_{2}^{A}$ display similar temperature 
(a) Change in $\mathrm{k}$ with temperature

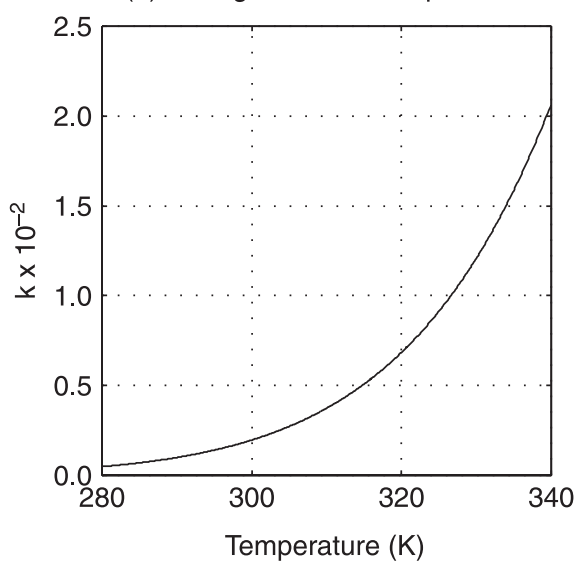

(b) Change in $\mathrm{K}$ with temperature

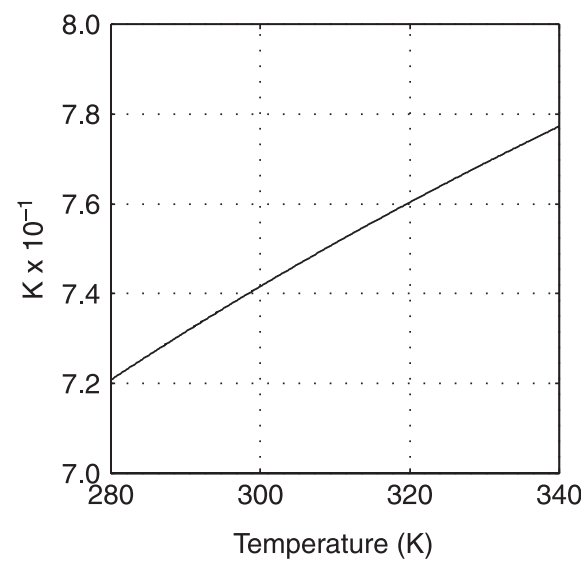

Figure 3. The change in the rate parameters $k$ and $K$ as the temperature is varied. $k$ is given by the Arrhenius equation (26) and $K$ is given by equation (27). Parameters are as follows: $R=8.31 \mathrm{~J} \mathrm{~K}^{-1} \mathrm{~mol}^{-1}, \quad E=50 \times 10^{3} \mathrm{~J} \mathrm{~mol}^{-1}, \quad B=10^{6} \mathrm{M}^{-1} \mathrm{~s}^{-1}, \quad E_{1}=50 \times 10^{3} \mathrm{~J} \mathrm{~mol}^{-1}$, $B_{1}=0.95 \times 10^{6} \mathrm{M}^{-1} \mathrm{~s}^{-1}, E_{-1}=51 \times 10^{3} \mathrm{~J} \mathrm{~mol}^{-1}, B_{-1}=1.05 \times 10^{6} \mathrm{M}^{-1} \mathrm{~s}^{-1}, E_{2}=49 \times 10^{3} \mathrm{~J} \mathrm{~mol}^{-1}$ and $B_{2}=1.00 \times 10^{3} \mathrm{M}^{-1} \mathrm{~s}^{-1}$.

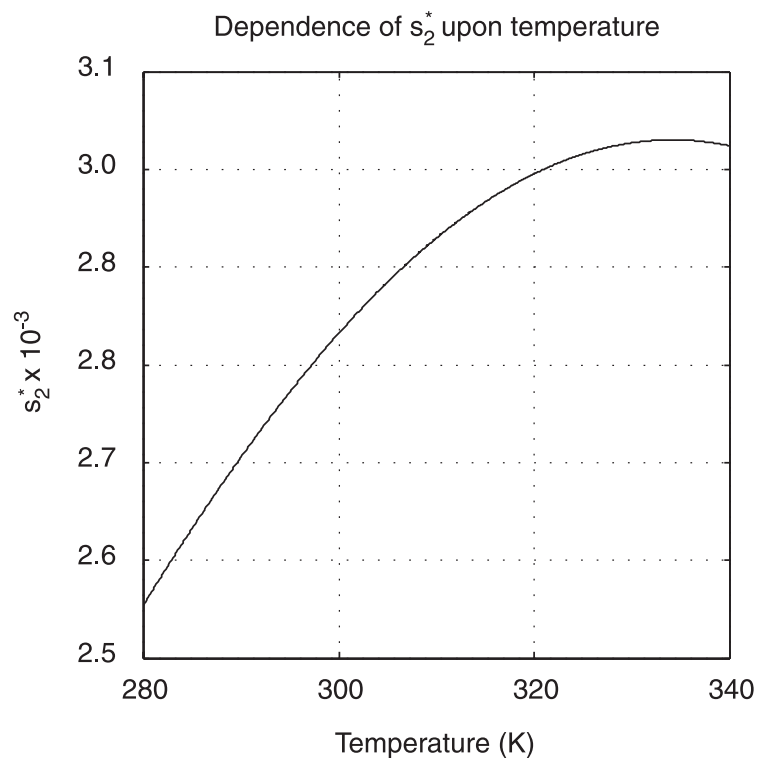

Figure 4. The temperature dependence of $s_{2}^{*}$ for the non-reversible reaction given by equation (1). $s_{2}^{*}$ is given by equation (13). The rate constants are assumed to display Arrhenius-type temperature dependencies and the active enzyme concentrations display a bell-shaped temperature dependence. Parameters are as follows: $\mu_{1}=305$, $\mu_{2}=308, \quad \sigma_{1}=20.0, \quad \sigma_{2}=18.0, \quad M_{1}=1.8 \times 10^{-4}, \quad M_{2}=2.0 \times 10^{-4}, R=8.31 \mathrm{~J} \mathrm{~K}^{-1} \mathrm{~mol}^{-1}, \quad E_{1}=5.00 \times$ $10^{4} \mathrm{~J} \mathrm{~mol}^{-1}, A_{1}=0.95 \times 10^{6} \mathrm{~s}^{-1}, E_{-1}=5.10 \times 10^{4} \mathrm{~J} \mathrm{~mol}^{-1}, A_{-1}=1.05 \times 10^{6} \mathrm{~s}^{-1}, E_{2}=4.90 \times 10^{4} \mathrm{~J} \mathrm{~mol}^{-1}$, $A_{2}=1.00 \times 10^{3} \mathrm{~s}^{-1}, E_{3}=5.10 \times 10^{4} \mathrm{~J} \mathrm{~mol}^{-1}, A_{3}=0.95 \times 10^{6} \mathrm{~s}^{-1}, E_{-3}=5.12 \times 10^{4} \mathrm{~J} \mathrm{~mol}^{-1}, A_{-3}=1.10 \times$ $10^{6} \mathrm{~s}^{-1}, E_{4}=4.93 \times 10^{4} \mathrm{~J} \mathrm{~mol}^{-1}$, and $A_{4}=1.00 \times 10^{2} \mathrm{~s}^{-1}$. 
dependencies. In particular, it should be noted that although $k_{2}$ and $k_{4}$ are highly temperature dependent, the resulting steady state value of $S_{2}$ displays a much weaker temperature dependence. In this way, we suggest a mechanism for achieving temperature regulation: suppose that we may write, for example,

$$
A_{i}=f_{i}(T, \mathbf{x}),
$$

where $f_{i}$ denotes the dependence of $A_{i}$ upon temperature, $T$, and upon a vector, $\mathbf{x}$, of other physiological parameters. Then we can write

$$
\frac{A_{i}}{A_{j}}=\frac{f_{i}(T, \mathbf{x})}{f_{j}(T, \mathbf{x})}=f_{i, j}(T, \mathbf{x}) .
$$

Our mathematical analysis suggests that the steady state concentration of the second substrate $S_{2}$ can be described as follows:

$$
s_{2}^{*}=\Theta\left[f_{2,1}(T, \mathbf{x})\right],
$$

for some function $\Theta$. This analysis holds under certain parameter constraints-whilst the Michaelis-Menten constants display weak temperature dependence-and in both the nonreversible and reversible bienzyme systems. In this case

$$
\frac{\mathrm{d} s_{2}^{*}}{\mathrm{~d} T}=\Theta^{\prime}\left[f_{2,1}(T, \mathbf{x})\right] \frac{\partial f_{2,1}(T)}{\partial T} .
$$

If the ratio of the $A_{i}\left(A_{i}^{\prime}\right)$ involved displays little temperature dependence we will have $\left|\partial f_{2,1} / \partial T\right| \ll 1$. Hence $\left(\mathrm{d} s_{2}^{*} / \mathrm{d} t\right) \approx 0$ for suitably smooth functions $\Theta$ and we see that $s_{2}^{*}$ will only display a weak temperature dependence. It is in this way that we suggest a possible mechanism for temperature dependence: that is, if certain relationships between the reaction schemes and temperature dependencies of individual enzyme activities are satisfied. We note that if the temperature of a system is changed then the results of section 2 only hold once the system has reached its new steady state.

\section{Chains of enzyme reactions}

In the previous section, we considered bienzyme modules: now we extend this analysis to consider chains of enzyme-facilitated reactions. As before, we will consider both reversible and non-reversible reactions.

\subsection{An irreversible enzyme chain}

Consider the following irreversible sequence of reactions:

$$
\begin{array}{ccccc}
S_{1}+E_{1} & \underset{k_{-1}}{\stackrel{k_{1}}{\rightleftharpoons}} & C_{1} & \stackrel{k_{2}}{\longrightarrow} & S_{2}+E_{1}, \\
S_{2}+E_{2} & \stackrel{k_{3}}{\underset{k_{-3}}{\rightleftharpoons}} & C_{2} & \stackrel{k_{4}}{\longrightarrow} & S_{3}+E_{2}, \\
\vdots & \vdots & \vdots & \vdots & \vdots \\
S_{n}+E_{n} & \underset{k-(2 n-1)}{\stackrel{k_{2 n-1}}{\rightleftharpoons}} & C_{n} & \stackrel{k_{2 n}}{\longrightarrow} & P+E_{n} .
\end{array}
$$


Using the Law of Mass Action, the module can be described by the chemical equations shown below:

$$
\begin{gathered}
\frac{\mathrm{d} s_{i}}{\mathrm{~d} t}=-k_{2 i-1} s_{i} e_{i}+k_{-(2 i-1)} c_{i}+k_{2(i-1)} c_{i-1}, \\
\frac{\mathrm{d} c_{i}}{\mathrm{~d} t}=k_{2 i-1} s_{i} e_{i}-\left(k_{-(2 i-1)}+k_{2 i}\right) c_{i}, \\
\frac{\mathrm{d} e_{i}}{\mathrm{~d} t}=-k_{2 i-1} s_{i} e_{i}+\left(k_{-(2 i-1)}+k_{2 i}\right) c_{i}, \\
\frac{\mathrm{d} p}{\mathrm{~d} t}=k_{2 n} c_{n},
\end{gathered}
$$

where, unless otherwise explicitly stated, the equations for reactant $i$ hold for $i=1,2, \ldots, n$. Following along exactly the same lines as the analysis for bienzyme systems, and assuming that the concentration of $S_{1}$ is fixed by other enzyme systems that we will not explicitly model, we have

$$
c_{i}(t)+e_{i}(t)=e_{i}^{A} \quad \text { for } \quad i=1,2, \ldots, n .
$$

Assuming that the system is in a steady state,

$$
c_{i}=\frac{e_{i}^{A} s_{i}}{K_{i}+s_{i}} \quad \text { for } \quad i=1,2, \ldots, n,
$$

where

$$
K_{i}=\frac{k_{-(2 i-1)}+k_{2 i}}{k_{2 i-1}} \quad \text { for } \quad i=1,2, \ldots, n .
$$

Hence the steady state solution for $S_{i}$ satisfies the equation

$$
\frac{A_{i-1}}{A_{i}}\left(K_{i}+s_{i}^{*}\right) s_{i-1}^{*}-s_{i}^{*}\left(K_{i-1}+s_{i-1}^{*}\right)=0,
$$

where $A_{i}=k_{2 i} e_{i}^{A}$ for $i=1,2, \ldots, n$. Rearranging the above gives $s_{i}^{*}$ in terms of $s_{i-1}^{*}$ :

$$
s_{i}^{*}=\frac{K_{i}}{\frac{A_{i}}{A_{i-1}}\left[\frac{K_{i-1}}{s_{i-1}}+1\right]-1} .
$$

The steady state concentration of substrate $i$ is therefore given by

$$
s_{i}^{*}=\frac{K_{i}}{\frac{A_{i}}{A_{1}}\left[\frac{K_{1}}{s_{1}^{0}}+1\right]-1} .
$$

Proof of the above is outlined in Appendix B. 
Making the same assumption as in section 2.1, that is, $s_{1}^{0} \gg K_{1}$, the approximate stationary solution for $S_{i}$ can be written

$$
s_{i}^{*}=\frac{K_{i}}{\frac{A_{i}}{A_{1}}-1} \quad \text { for } \quad i=2,3, \ldots, n .
$$

To consider temperature dependence we use the same notation as previously:

$$
s_{n}^{*}=\Theta_{n}\left[f_{n, 1}(T) g_{n, 1}(\mathbf{x})\right],
$$

for some function $\Theta_{n}$. Using a similar argument to before, we see that if the ratio of $A_{n} / A_{1}$ displays little temperature dependence (so that $f_{n, 1}(T)$ is approximately constant), the steady state concentration of $S_{n}$ will display only a weak temperature dependence: it does not matter that the individual components involved in the reaction may be highly temperature dependent.

\subsection{A reversible enzyme chain}

Consider the following reversible sequence of reactions:

$$
\begin{array}{ccccc}
S_{1}+E_{1} & \underset{k_{-1}}{\stackrel{k_{1}}{\rightleftharpoons}} & C_{1} & \stackrel{k_{2}}{\longrightarrow} & S_{2}+E_{1}, \\
S_{2}+E_{1}^{\prime} & \underset{k_{-3}^{\prime}}{\stackrel{k_{3}^{\prime}}{\rightleftharpoons}} & C_{2}^{\prime} & \stackrel{k_{2}^{\prime}}{\longrightarrow} & S_{1}+E_{2}^{\prime}, \\
S_{2}+E_{2} & \underset{k_{-3}}{\stackrel{k_{3}}{\rightleftharpoons}} & C_{2} & \stackrel{k_{4}}{\longrightarrow} & S_{3}+E_{2}, \\
\vdots & \vdots & \vdots & \vdots & \vdots \\
S_{n}+E_{n} & \underset{k_{-(2 n-1)}}{\stackrel{k_{2 n-1}}{\rightleftharpoons}} & C_{n} & \stackrel{k_{2 n}}{\longrightarrow} & P+E_{n}, \\
P+E_{n}^{\prime} & \underset{k_{2 n+1}^{\prime}}{\stackrel{k_{-(2 n+1)}^{\prime}}{\rightleftharpoons}} & C_{n}^{\prime} & \stackrel{k_{2 n}^{\prime}}{\longrightarrow} & S_{n}+E_{n}^{\prime} .
\end{array}
$$

Using the Law of Mass Action, the sequence can be described by the following set of equations:

$$
\begin{gathered}
\frac{\mathrm{d} s_{1}}{\mathrm{~d} t}=-k_{1} s_{1} e_{1}+k_{-1} c_{1}+k_{2}^{\prime} c_{1}^{\prime}, \\
\frac{\mathrm{d} s_{i}}{\mathrm{~d} t}=-k_{2 i-1} s_{i} e_{i}+k_{-(2 i-1)} c_{i}+k_{2(i-1)} c_{i-1} \\
-k_{2(i-1)+1}^{\prime} s_{i} e_{i-1}^{\prime}+k_{-(2(i-1)+1)}^{\prime} c_{i-1}^{\prime}+k_{2 i}^{\prime} c_{i}^{\prime}, \\
\frac{\mathrm{d} c_{i}}{\mathrm{~d} t}=k_{2 i-1} s_{i} e_{i}-\left(k_{-(2 i-1)}+k_{2 i}\right) c_{i}, \\
\frac{\mathrm{d} e_{i}}{\mathrm{~d} t}=-k_{2 i-1} s_{i} e_{i}+\left(k_{-(2 i-1)}+k_{2 i}\right) c_{i},
\end{gathered}
$$




$$
\begin{gathered}
\frac{\mathrm{d} c_{i}^{\prime}}{\mathrm{d} t}=k_{2 i+1}^{\prime} s_{i+1} e_{i}^{\prime}-\left(k_{-(2 i+1)}+k_{2 i}^{\prime}\right) c_{i}^{\prime}, \\
\frac{\mathrm{d} e_{i}^{\prime}}{\mathrm{d} t}=-k_{2 i+1}^{\prime} s_{i+1} e_{i}^{\prime}+\left(k_{-(2 i+1)}+k_{2 i}^{\prime}\right) c_{i}^{\prime}, \\
\frac{\mathrm{d} p}{\mathrm{~d} t}=k_{2 n} c_{n},
\end{gathered}
$$

where, unless otherwise explicitly stated, the equations for reactant $i$ hold for $i=1,2, \ldots, n$. We have

$$
c_{i}(t)+e_{i}(t)=e_{i}^{A} \quad \text { and } \quad c_{i}^{\prime}(t)+e_{i}^{\prime}(t)=e_{i}^{\prime A} \quad \text { for } \quad i=1,2, \ldots, n .
$$

Assuming that the system is in a steady state we have, for $i=1,2, \ldots, n$ :

$$
c_{i}=\frac{e_{i}^{A} s_{i}}{K_{i}+s_{i}} \quad \text { and } \quad c_{i}^{\prime}=\frac{e_{i}^{\prime A} s_{i+1}}{K_{i+1}^{\prime}+s_{i+1}},
$$

where

$$
K_{i}=\frac{k_{-(2 i-1)}+k_{2 i}}{k_{2 i-1}}, \quad \text { and } \quad K_{i+1}^{\prime}=\frac{k_{-(2 i+1)}^{\prime}+k_{2 i}^{\prime}}{k_{2 i+1}^{\prime}} .
$$

In a similar manner as before, for $i=2,3, \ldots, n$ :

$$
\begin{aligned}
0= & -k_{2 i} c_{i}+k_{2(i-1)} c_{i-1}-k_{2(i-1)}^{\prime} c_{i-1}^{\prime}+k_{2 i}^{\prime} c_{i}^{\prime}, \\
= & -k_{2 i} e_{i}^{A} \frac{s_{i}}{K_{i}+s_{i}}+k_{2(i-1)} e_{i-1}^{A} \frac{s_{i-1}}{K_{i-1}+s_{i-1}}-k_{2(i-1)}^{\prime} e_{i-1}^{\prime A} \frac{s_{i}}{K_{i}^{\prime}+s_{i}} \\
& +k_{2 i}^{\prime} e_{i}^{\prime A} \frac{s_{i+1}}{K_{i+1}^{\prime}+s_{i+1}} .
\end{aligned}
$$

Letting

$$
\lambda_{i}=A_{i} \frac{s_{i}}{K_{i}+s_{i}}-A_{i-1} \frac{s_{i-1}}{K_{i-1}+s_{i-1}}+A_{i-1}^{\prime} \frac{s_{i}}{K_{i}^{\prime}+s_{i}},
$$

where

$$
A_{i}=k_{2 i} e_{i}^{A} \quad \text { and } \quad A_{i}^{\prime}=k_{2 i}^{\prime} e_{i}^{\prime A},
$$

the steady state solution, $s_{i+1}^{*}$ is given in terms of $s_{i}^{*}$ by

$$
s_{i+1}^{*}=\frac{K_{i+1}^{\prime}}{\frac{A_{i}^{\prime}}{\lambda_{i}}-1} .
$$


The steady state concentration of substrate $i$ is therefore given by

$$
s_{i+1}^{*}=\frac{K_{i+1}^{\prime}}{\frac{A_{i}^{\prime}}{A_{i}}\left[\frac{K_{i}}{s_{i}^{*}}+1\right]-1} .
$$

Proof of the above is outlined in Appendix C.

From previous results, with $s_{1}^{0} \gg K_{1}$,

$$
s_{2}^{*} \approx \frac{K_{2}^{\prime}}{\frac{A_{1}^{\prime}}{A_{1}}-1} .
$$

In this case, we note that

$$
s_{i+1}^{*}=f\left(\frac{A_{1}^{\prime}}{A_{1}}, \ldots, \frac{A_{i}^{\prime}}{A_{i}}\right) .
$$

Using similar notation as before, if

$$
\frac{A_{i}^{\prime}}{A_{i}}=\frac{\bar{f}_{i}^{\prime}(T, \mathbf{x})}{\tilde{f}_{i}(T, \mathbf{x})}=f_{i}(T, \mathbf{x}),
$$

then

$$
s_{n}^{*}=\Theta\left[f_{1}(T, \mathbf{x}), \ldots, f_{n-1}(T, \mathbf{x})\right] .
$$

In this way, we see that in contrast to the non-reversible system, the steady state concentration of $S_{n}$ is dependent on the temperature dependence of each of the individual reactions: the longer the chain the higher the temperature dependence is likely to be.

\section{Conclusions}

In this paper, we used standard reaction kinetics and associated analysis to investigate the temperature dependence of bienzyme modules and long enzymatic chains. Initial models showed that under certain conditions the stationary states of bienzyme modules may be only weakly influenced by temperature, despite strong temperature dependence of the separate reactions, if the reaction rates and enzyme activities show similar temperature dependence. Subsequent models suggest a mechanism for temperature compensation in metabolic chains: non-reversible chains showed a temperature dependence on only the first and final components of the chain whilst reversible chains were shown to display a dependence on every component.

It is important to note that in each case examined here, the system requires a transient period following each temperature change until it reaches a new steady state. The analysis presented here is not valid until the system reaches this steady state. It is also important to note that the results only hold for proteins (substrates) which either do not denature significantly over the temperature range of interest, or which are not labile to temperature. 


\subsection{Example}

We now illustrate our theoretical findings with some experimental results from Paramecium caudatum.

P. caudatum is a unicellular organism with the ability to survive over a wide temperature range. Movement is controlled by the beating of cilia arranged around the cell surface and the biomolecular system understood to control cilia beat frequency in P. caudatum is presented in figure 5. This scheme is based on the data presented in several papers $[4,5,21,24,26]$. We note that the scheme shown in figure 5 includes several of the bienzyme modules discussed in section 2. Experimental data suggests that the individual $Q_{10}$ for some of the individual reactions involved in this scheme are as follows: Protein kinase, $Q_{10} \approx 2.3$ [6]; Phosphatase, $Q_{10} \approx 5.0$ [35]; AC, $Q_{10} \approx 2.5-3.0$ [39].

The dependence of $P$. caudatum motion velocity upon temperature has been measured and the results are shown in figure 6: for more details of the experimental procedures used in gathering these results see Appendix A. An estimate of the temperature coefficient, $Q_{10}$, was obtained by considering cilia beat frequency, which itself determines $P$. caudatum movement velocity. From figure 6 , we estimate $Q_{10}=1.624$, thereby illustrating that the metabolic scheme consisting of a combination of the bi-enzyme modules described above has a temperature dependence that is lower than the temperature dependence of the individual modules. This experimental result supports the theoretical predictions reported here.

\subsection{Discussion}

It could be inferred that evolution has been directed to make the temperature dependencies of enzyme activities close to one another, hence providing a mechanism for compensating against the effects of temperature on some of the main cellular processes. At the same time the dynamic behavior of the considered modules making up these processes remains strongly influenced by the temperature. These results are valid for a range of bienzyme systems: for cGMP metabolism; acetylation; adenylation; and the uridilation of proteins.

The results obtained here can be used in experimental studies of the temperature effects upon living biological systems. Our studies suggest that the effect of temperature upon sophisticated biological processes is determined not only by the presence or absence of

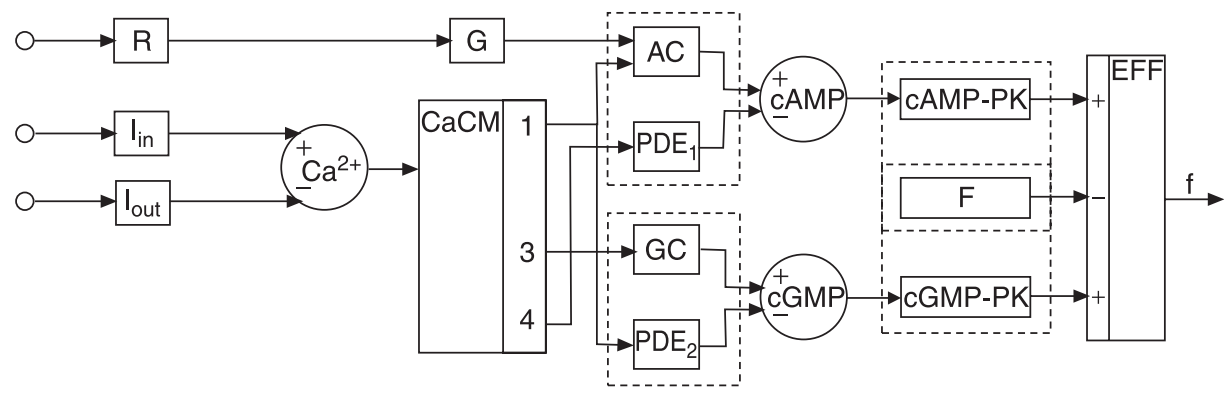

Figure 5. Cilia beat frequency controlling system in $P$. caudatum. R-receptor, G-G protein, CaCM-

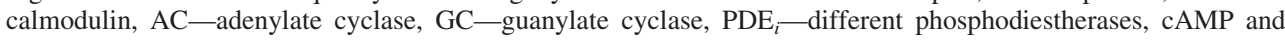
cGMP cyclic nucleotides, cAMP-PK and cGMP-PK - protein kinases, F-phosphoproteinphosphotase, EFF group of effector proteins, $\mathrm{f}$ - cilia beating frequency, $\mathrm{I}_{\mathrm{in}}, \mathrm{I}_{\mathrm{out}}$ - passive and active ion currents. The dashed boxes denote the bienzyme modules. 


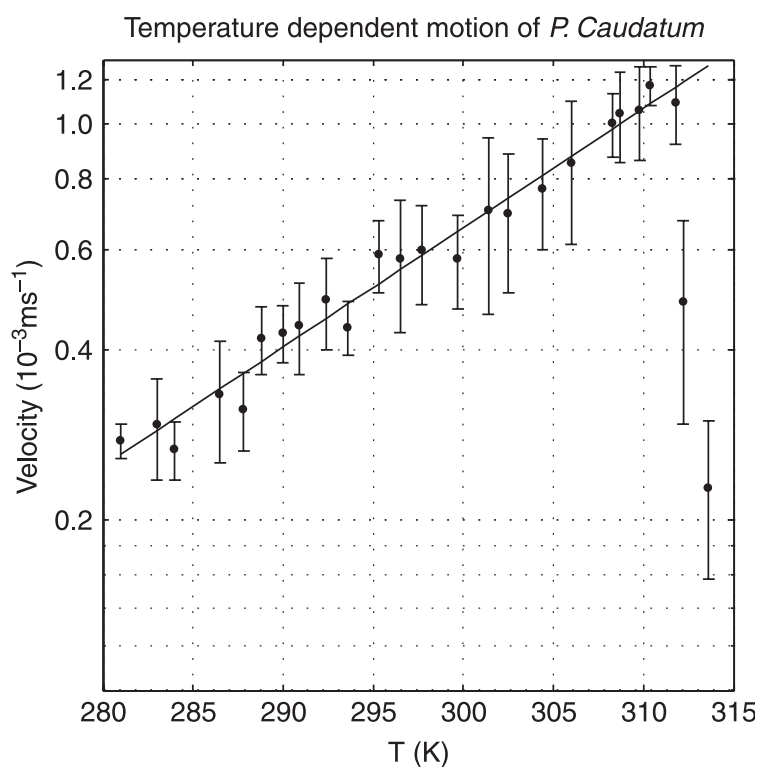

Figure 6. The motion velocity of Paramecium caudatum over a range of temperatures. Here, $Q_{10}$ is calculated to be approximately 1.624 for all points. See Appendix A for more details.

enzymes, but also strongly depends on the structure of the connections between the reactions. In the majority of cases, enzymes control metabolite concentrations in eukaryotes and most of the enzyme reactions are strongly dependent on temperature. Some of the metabolites are the signal agents: for example, cyclic nucleotides, hormones, calcium ions amongst others.

It is important to notice that the condition of similarity of enzymatic activities may not be valid in the whole physiological temperature range. This in particular can be true in the case of cold-blooded animals, due to the structural differences of enzymes forming the bienzyme module. To adapt to wider temperature ranges evolution might have selected two or more isoforms of the same enzyme with different temperature dependencies [22]. This type of adaptation is connected with the change in gene expression; therefore it is quite slow and not considered here.

Having said that, the body temperature of cold-blooded animals can vary over a wide range, whereas in the case of warm-blooded animals the temperature only changes by a few degrees, yet animals remain alive despite these changes. We speculate that the usage of bienzyme modules and non-reversible enzymatic reactions allows for some temperature compensation: it is therefore possible that a system that has highly temperature dependent components is almost temperature independent. We speculate that on time scales shorter than the times required for changes in gene expression, the usage of such modules is sufficient to compensate for the strong temperature dependence of individual enzymes.

\section{Acknowledgements}

REB would like to thank the London Mathematical Society for a Small Collaborative Grant to visit the Laboratory of Bionics and Biophysics, Kazan State University, Lloyds Tercentenary Foundation for a Lloyds Tercentenary Foundation Fellowship, Research 
Councils UK for an RCUK Academic Fellowship in Mathematical Biology and St Hugh's College, Oxford for a Junior Research Fellowship. REB would also like to thank the Max Planck Institute for Mathematics in the Sciences for a visiting position. NVK and AIS would like to thank the Russian Foundation for Basic Research and the Russian government for a Leading Scientific School grant. NVV gratefully acknowledges support from ORS, The Hill Foundation for a Hill Foundation Scholarship and The Wellcome Trust for a Prize studentship (070417). The authors would also like to thank Santiago Schnell for helpful comments on the manuscript.

\section{References}

[1] Alberts, B., Johnson, A., Lewis, J., Raff, M., Roberts, K. and Walter, P., 2002, Molecular Biology of the Cell, 4th ed. (New York).

[2] Allen, T.J.A. and Micala, G., 1998, Effects of temperature on human L-type cardiac $\mathrm{Ca}^{+}$channels expressed in Xenopus oocytes, European Journal of Physiology, 436, 238-247.

[3] Bergamasco, R., Bassetti, F.J., Moraes, F.F. and Zanin, M., 2000, Characterization of free and immobilized invertase regarding activity and energy of activation, Brazilian Journal of Chemical and Engineering, 17(4-7), $873-880$.

[4] Bonini, N.M., Gustin, M.C. and Nelson, D.L., 1986, Regulation of ciliary motion by membrane potential in Paramecium: a role for cyclic AMP, Cell Motility and the Cytoskeleton, 6(3), 256-272.

[5] Bonini, N.M. and Nelson, D.L., 1988, Differential regulation of Paramecium ciliary motility by cAMP and cGMP, The Journal of Cell Biology, 106, 1615-1623.

[6] Brooks, S.P. and Storey, K.B., 1996, Protein kinase involvement in land snail aestivation and anoxia: protein kinase A kinetic properties and changes in second messenger compounds during depressed metabolism, Molecular and Cellular Biochemistry, 156(2), 153-161.

[7] Chaplin, M.F. and Bucke, C., 1990, Enzyme Technology (Cambridge: Cambridge University Press).

[8] Chaui-Berlinck, J.G., Monteir, L.H.A., Navas, C.A. and Bicudo, J.E.P.W., 2002, Temperature effects on energy metabolism: a dynamic system analysis, Proceedings of the Royal Society of London B, 269, 15-19.

[9] Chaui-Berlinck, J.G., Navas, C.A., Monteir, L.H.A. and Bicudo, J.E.P.W., 2004, Temperature effects of a whole metabolic reaction cannot be inferred from its components, Proceedings of the Royal Society of London B, 271, $1415-1419$.

[10] Cornish-Bowden, A., 1995, Fundamentals of Enzyme Kinetics, 2nd ed. (Londo: Portland Press).

[11] Cornish-Bowden, A., 1999, Enzyme kinetics from a metabolic perspective, Biochemical Society Transactions, 27(2), 281-284.

[12] Daniel, R.M., Dines, M. and Petach, H.H., 1996, The denaturation and degradation of stable enzymes at high temperatures, Biochemical Journal, 317(1), 1-11.

[13] Dixon, M. and Webb, E.C., 1979, Enzymes, 3rd ed. (London: Longman).

[14] Feller, G. and Gerday, C., 1997, Psychrophilic enzymes: molecular basis of cold adaptation, Cellular and Molecular Life Sciences, 53, 830-841.

[15] Finn, F.M., Montibeller, J.A., Ushikima, Y. and Hofmann, K., 1975, Adenylate cyclase system of bovine adrenal plasma membranes, Journal of Biological Chemistry, 250(4), 1186-1192.

[16] Gage, P.W. and McBurney, R.N., 1975, Effects of membrane potential, temperature and neostigmine on the conductance charge caused by a quantum of acetycholine at the toad neuromuscular junction, Journal of Physiology, 244, 385-407.

[17] Georlette, D., Blaise, V., Collins, T., D'Amico, S., Gratia, E., Hoyoux, A., Marx, J.-C., Sonan, G., Feller, G. and Gerday, C., 2004, Some like it cold: biocatalysis at low temperatures, FEMS Microbiology Reviews, 28(1), $25-42$.

[18] Gribble, F.M., Loussouarn, G., Tucker, S.J., Zhao, C., Nichols, C.G. and Ashcroft, F.M., 2000, A novel method for measurement of submembrane ATP concentration, Journal of Biological Chemistry, 275(39), 3004630049.

[19] Harper, A.A., Shelton, J.R. and Watt, P.W., 1989, The temperature dependence of the time course of growth and decay of miniature end-plate currents in carp extraocular muscle following thermal acclimation, Journal of Experimental Biology, 147, 237-248.

[20] Head, S.D., 1983, Temperature and end-plate currents in rat diaphragm, Journal of Physiology, 334, 441-459.

[21] Hennessey, T., Machemer, H. and Nelson, D.L., 1985, Injected cyclic AMP increases ciliary beat frequency in conjunction with membrane hyperpolarisation, European Journal of Cell Biology, 36(2), 153-156.

[22] Hochachka, P.W. and Somero, G.N., 1984, Biochemical Adaptation (Princeton: Princeton University Press).

[23] Hubbard, J.I., Jones, S.F. and Landau, E.M., 1971, The effect of temperature change upon transmitter release, facilitation and post-titanic potentiation, Journal of Physiology, 216, 596-609. 
[24] Kinosita, H. and Murakami, A., 1967, Control of ciliary motion, Physiology Review, 47(1), 53-82.

[25] Kiyohara, T., Hirata, H., Hori, T. and Akaike, N., 1990, Hypothalmic warm-sensitive neurons possess a tetrodotoxin-sensitive sodium channel with a high $Q_{10}$, Neuroscience Research, 8(1), 48-53.

[26] Klumpp, S. and Schultz, E., 1982, Characterisation of a $\mathrm{Ca}^{2+}$-dependent guanylate cyclase in the excitable ciliary membrane from Paramecium, European Journal of Biochemistry, 124, 317-324.

[27] Kordas, M., 1972, An atempt at an analysis of the factors determining the time course of the end-plate current II. Temperature, Journal of Physiology, 224, 346-349.

[28] Lee, J.H. and Timmons, R.B., 1976, Absolute rate parameteres for the reaction of ground state atomic oxygen with dimethyl sulfide and episulfide, Journal of Chemical Physics, 64(1), 300-305.

[29] Lin, M.C., Salomon, Y., Rendell, M. and Rodbell, M., 1975, The hepatic adenylate cyclase system. II. Substrate binding and utilisation and the effects of magnesium ion and $\mathrm{pH}$, Journal of Biological Chemistry, 250(11), 4246-4252 June.

[30] Londesborough, J. and Varimo, K., 1979, The temperature-dependence of adenylate cyclase from baker's yeast, Biochemical Journal, 181(3), 539-543.

[31] MacDonald, J.A. and Storey, K.B., 1988, cAMP-dependent protein kinase from brown adipose tissue: temperature effects on kinetic properties and enzyme role in hibernating ground squirrels, Journal of Comparative Physiology, 168(7), 513-525.

[32] MacDonald, J.A. and Storey, K.B., 1999, Regulation of ground squirrel $\mathrm{Na}^{+} \mathrm{K}^{+}$-ATPase activity by reversible phosphrylation during hibernation, Biochemical and Biophysical Research Communications, 254, 424-429.

[33] MacDonald, J.A. and Storey, K.B., 2005, Temperature and phosphate effects on allosteric phenomena of phosphofructokinase from a hibernating ground squirrel (Spermophilus lateralis), FEBS Journal, 272, $120-128$.

[34] Magazanik, L.G. and Vyskocit, F., 1975, The effect of temperature on desensitisation kinetics at the postsynaptic membrane of the frog muscle fibre, Journal of Physiology, 249(2), 285-300.

[35] Mitsui, T., Kitazawa, T. and Ikebe, I., 1994, Correlation between high temperature dependence of smooth muscle myosin light chain phosphatase activity and muscle relaxation rate, Journal of Biological Chemistry, 25, 5842-5848.

[36] Ravishankara, A.R., Eisele, F.L. and Wine, P.H., 1983, The kinetics of the reaction of OH with CIO, Journal of Chemical Physics, 78(3), 1140-1144.

[37] Robertson, B. and Wann, K.T., 1984, The effect of temperature on the growth decay time of miniature end-plate currents in the mouse diaphragm, Brain Research, 294, 346-349.

[38] Ruff, R.L., 1999, Effects of temperature on slow and fast inactivation of rat skeletal muscle $\mathrm{Na}^{+}$channels, American Journal of Physiology Cell Physiology, 277, C937-C947.

[39] Scott, W.A., 1976, Adenosine $3^{\prime}: 5^{\prime}$-cyclic monophosphate deficiency in Neurospora crassa, Proceedings of the National Academy of Sciences USA, 73(9), 2995-2999.

[40] Segerer, A.H., Burggraf, S., Fiala, G., Huber, G., Huber, R., Pley, U. and Stetter, K.O., 1993, Life in hot springs and hydrothermal vents, Origin of Life and Evolution of Biosphere, 23(1), 77-90.

[41] Sharp, K., 2001, Entropy-enthalpy compensation: fact or artifact?, Protein Sciences, 10(3), 661-667.

[42] Sheridan, P.P., Panasik, N., Coombs, J.M. and Brenchley, E., 2000, Approaches for deciphering the structural basis of low temperature enzyme activity, Biochimica et Biophysica Acta, 1543(2), 417-433.

[43] Stödeman, M. and Schwarz, P., 2003, Temperature dependence of the kinetics of the acylase hydrolysis reaction by differential stopped flow microcalorimetry, Analytical Biochemistry, 321, 1-7.

[44] Tiiska, A.J. and Lagerspetz, Y.H., 1994, Thermal acclimation, neuromusclar synaptic delay and miniature end-plate current decay in the frog Rana Temporaria, Journal of Experimental Biology, 187, 131-142.

\section{Appendix A: Paramecium caudatum}

Specimens of $P$. caudatum grown in hay infusion were isolated for experimentation in a solution of $1 \mathrm{mM} \mathrm{CaCl}_{2}, 1 \mathrm{mM} \mathrm{KCl}$ at $\mathrm{pH} 7.2$ (Tris- $\mathrm{HCl}$ ). A small amount of experimental solution with 5-7 specimens was placed in a chamber built of thin glass. To prevent vaporisation of solution the chamber was covered with coverglass. The bottom wall of the chamber is the wall of the thermostat. The temperature of the thermostatic fluid (water) was changed within the range $5-40^{\circ} \mathrm{C}$. The tolerance of temperature maintenance was $0.2^{\circ} \mathrm{C}$. Using a microscope and video camera, images of swimming $P$. caudatum cells were transferred onto a computer where specialist software processed the images and calculated the parameters of cell motion. The dependence of $P$. caudatum motion velocity upon temperature calculated by this method is shown in figure 6 . 


\section{Appendix B: Proof of result for irreversible enzyme chain}

The steady state concentration of substrate $i$ is given by

$$
s_{i}^{*}=\frac{K_{i}}{\frac{A_{i}}{A_{1}}\left[\frac{K_{1}}{s_{1}^{*}}+1\right]-1} .
$$

Proof. The proof can easily be established by induction: from section 2.1,

$$
s_{2}^{*}=\frac{K_{2}}{\frac{A_{2}}{A_{1}}\left[\frac{K_{1}}{s_{1}^{*}}+1\right]-1} .
$$

Hence equation (B1) holds for $i=2$. Suppose that equation (B1) holds for $i=2,3, \ldots, j$. In this case:

$$
\begin{aligned}
s_{j+1}^{*} & =\frac{K_{j+1}}{\frac{A_{j+1}}{A_{j}}\left[\frac{K_{j}}{s_{j}^{*}}+1\right]-1}, \\
& =\frac{K_{j+1}}{\frac{A_{j+1}}{A_{j}}\left[\frac{K_{j}}{K_{j}}\left\{\frac{A_{j}}{A_{1}}\left[\frac{K_{1}}{s_{1}^{*}}+1\right]-1\right\}+1\right]-1}, \\
& =\frac{K_{j+1}}{\frac{A_{j+1}}{A_{1}}\left[\frac{K_{1}}{s_{1}^{*}}+1\right]-1} .
\end{aligned}
$$

Hence equation (B1) holds for $\forall i=2,3, \ldots, n$ and the proof is complete.

\section{Appendix C: Proof of result for reversible enzyme chain}

The steady state concentration of substrate $i$ is given by

$$
s_{i+1}^{*}=\frac{K_{i+1}^{\prime}}{\frac{A_{i}^{\prime}}{A_{i}}\left[\frac{K_{i}}{s_{i}^{*}}+1\right]-1} .
$$

Proof. The proof can again easily be established by induction. From previous results:

$$
s_{2}^{*}=\frac{K_{2}^{\prime}}{\frac{A_{1}^{\prime}}{A_{1}}\left[\frac{K_{1}}{s_{1}^{*}}+1\right]-1},
$$

hence equation (C1) holds for $i=2$. Suppose that equation (C1) holds for $i=2,3, \ldots, j+1$. In this case:

$$
\begin{aligned}
\lambda_{j+1} & =A_{j+1} \frac{1}{\frac{K_{j+1}}{s_{j+1}^{*}}+1}-A_{j} \frac{1}{\frac{K_{j}}{s_{j}^{*}}-1}+A_{j}^{\prime} \frac{1}{\frac{K_{j+1}^{\prime}}{s_{j+1}^{*}}+1}, \\
& =A_{j+1} \frac{1}{\frac{K_{j+1}^{*}}{s_{j+1}^{*}}+1}-A_{j} \frac{1}{\frac{K_{j}}{s_{j}^{j}}-1}+A_{j}^{\prime} \frac{1}{\frac{K_{j+1}^{\prime}}{K_{j+1}^{\prime}}\left\{\frac{A_{j}^{\prime}}{A_{j}}\left[\frac{K_{j}}{s_{j}^{j}}+1\right]-1\right\}+1}, \\
& =A_{j+1} \frac{1}{\frac{K_{j+1}^{*}}{s_{j+1}^{*}}+1},
\end{aligned}
$$


which gives

$$
s_{j+2}^{*}=\frac{K_{j+2}^{\prime}}{\frac{A_{j+1}^{\prime}}{\lambda_{j+1}}-1}=\frac{K_{j+2}^{\prime}}{\frac{A_{j+1}^{\prime}}{A_{j+1}}\left[\frac{K_{j+1}}{s_{j+1}^{\prime}}+1\right]-1} .
$$

Hence equation $(\mathrm{C} 1)$ holds $\forall i=2,3, \ldots, n$ and the proof is complete. 


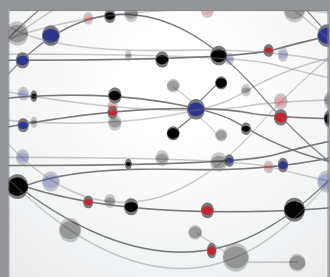

The Scientific World Journal
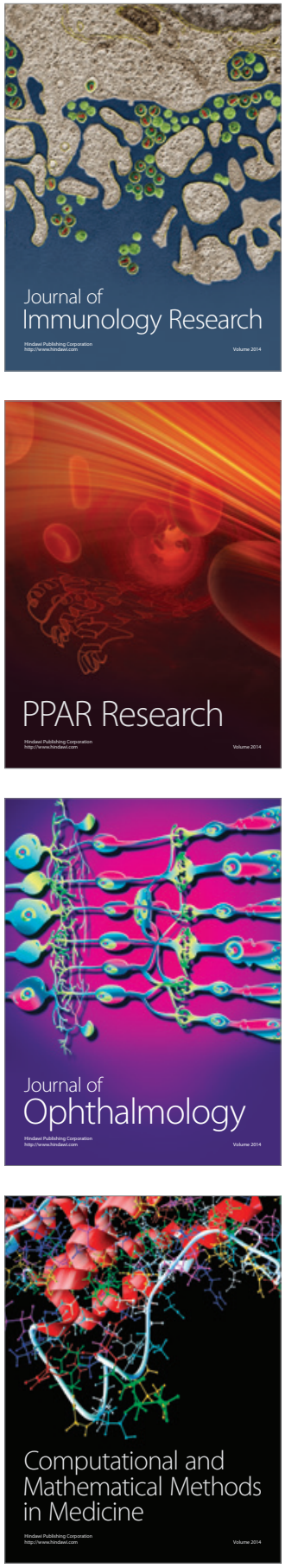

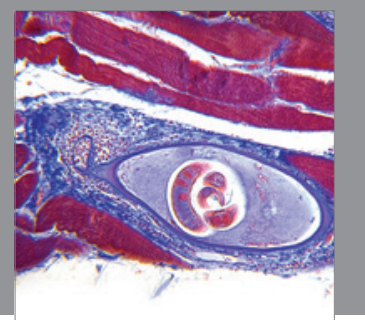

Gastroenterology

Research and Practice
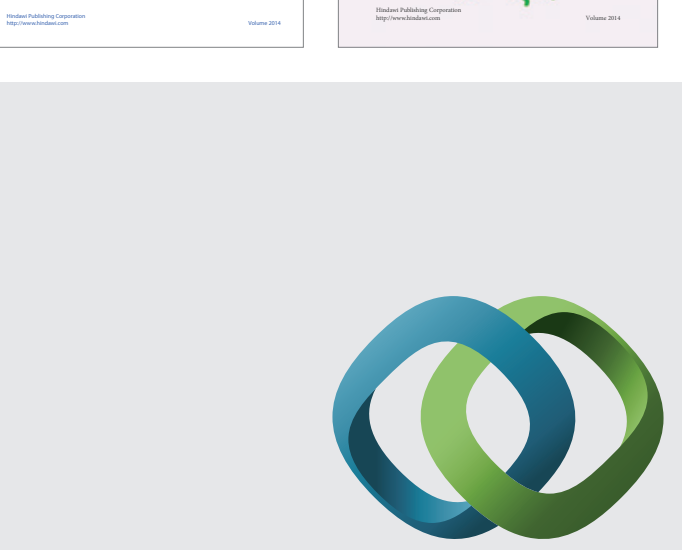

\section{Hindawi}

Submit your manuscripts at

http://www.hindawi.com
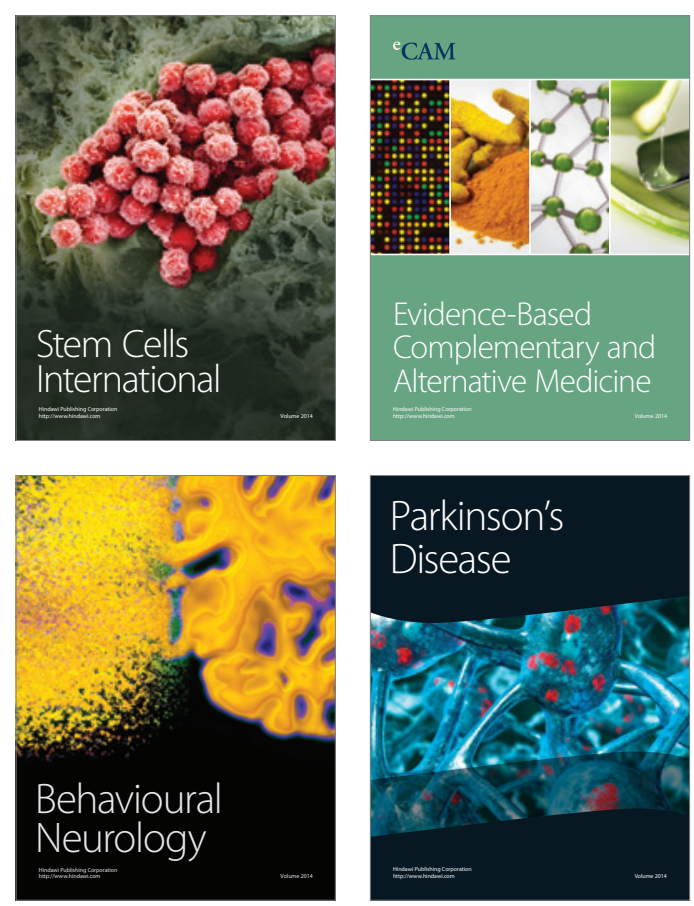

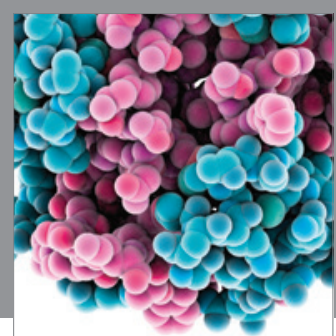

Journal of
Diabetes Research

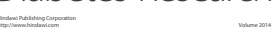

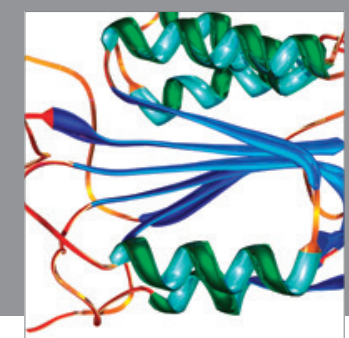

Disease Markers
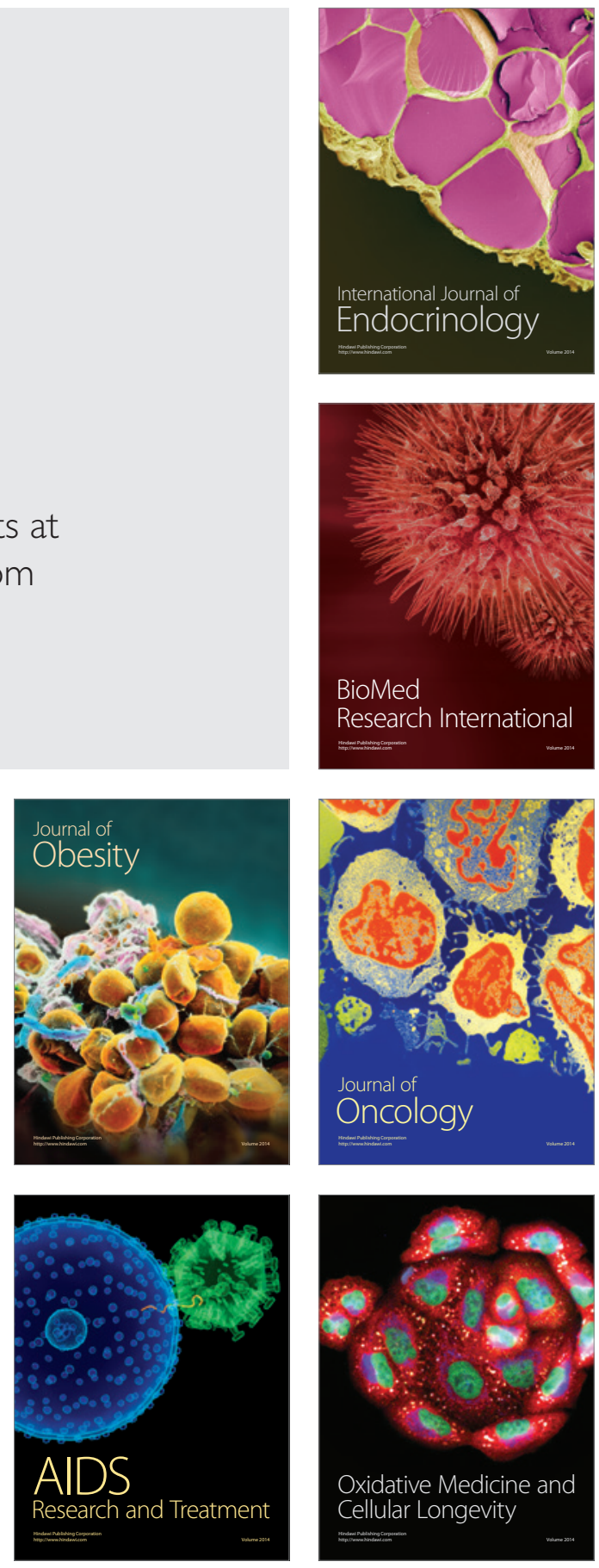